\title{
New Aspects of Input Shaping Control to Damp Oscillations of a Compliant Force Sensor
}

\author{
Amine Kamel, Friedrich Lange and Gerd Hirzinger \\ German Aerospace Center (DLR) \\ Institute of Robotics and Mechatronics \\ Oberpfaffenhofen, D-82234 Wessling, Germany \\ Friedrich.Langeedlr.de
}

\begin{abstract}
Compliance in robot mounted force/torque sensors is useful for soft mating of parts. However it generates nearly undamped oscillations when moving the end-effector in free space. In this paper, input shaping control is investigated to damp such unwanted flexible modes. We present a new design technique that creates long impulse sequences to adapt input shaping to systems with long sampling period and to compensate the resulting time delay. This makes the method feasible for industrial robots. In addition to the conventional input shaping which causes oscillations to stop only after applying the last impulse, we also minimize the quadratic control error until this time step is reached.
\end{abstract}

\section{INTRODUCTION}

Compliant force/torque sensors are useful for assembly tasks since they perform measurements and allow to give way to high-frequency motions of the part that has to be approached. This is fundamental for mating parts to moved objects, as in an assembly line. However, heavy end-effectors tend to oscillate as long as the tool is moved in free space. Such oscillatory behavior is critical in many robotic applications, especially for some tasks with high speed and precision requirements. Fig. 1 shows the setup of such a task - the assembly of wheels to a continuously moved car. Disturbances of the car motion are tolerated by a compliant end-effector (fig. 2) which, unfortunately, presents very poor damping. Input shaping also known as command shaping is one of the

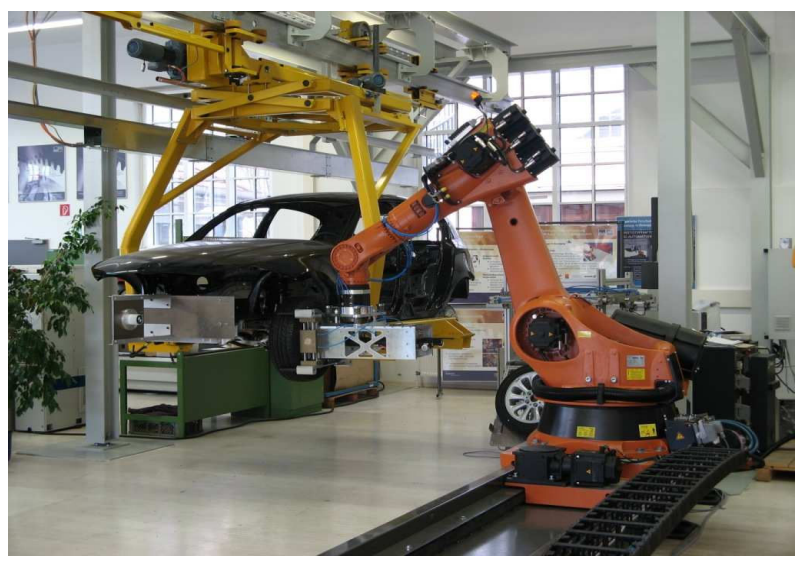

Fig. 1. Setup for wheel assembly

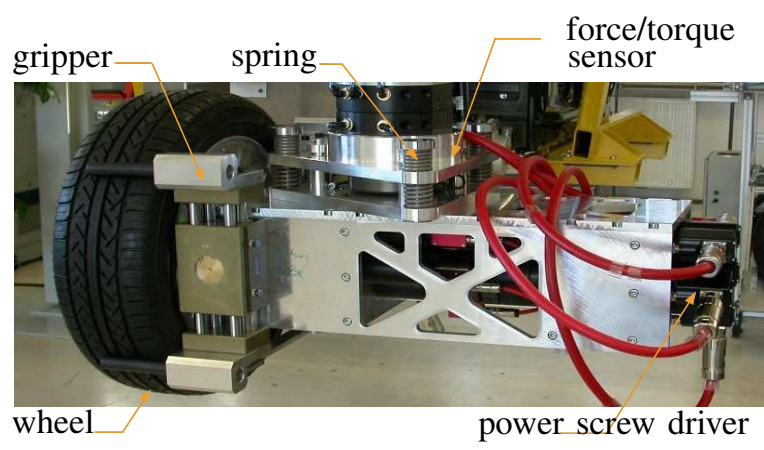

Fig. 2. Compliant end-effector with force/torque sensor in the center of the springs.

easiest successfully applied feedforward control techniques that have been designed to suppress residual vibrations occurring within speedy maneuvers. It consists of generating commands that move the system without vibration using some pre-knowledges about the plant. Smith [1] presented 1957 the posicast control as a first form of input shaping. It consists in generating two transient oscillations which cancel each other and lead to a non-oscillatory response. It has taken around 30 years till the first paper of input shaping was published by Singer and Seering [2]. System inputs were convolved to a sequence of impulses to generate adjusted command signals that eliminate the oscillatory dynamics. Since then many extensions of the method appeared: Various approaches like adding more impulses to the input shaper have been developed to achieve any order of robustness against parameter uncertainties (Singhose [3]). Furthermore, adaptive Input Shaping schemes were developed to keep the length of the impulse sequence to a minimum and to provide similar robustness ([4][5][6]). Many other approaches have shown that objectives related to input shaping can be reformulated as an optimization problem. This is based on the fact that designing an input shaper is nothing else but a particular FIR filter design problem([7]). Robertson, Kozak and Singhose discussed in [8] the minimization of several useful cost functions when designing digital shapers. The performance of these approaches is well established and has been demonstrated on many practical system such 
as spacecrafts (Tuttle und Seering [9]) and tower cranes (Singhose and Kim [10]).

The convolution of inputs with an impulse can be reduced to a shifting and scaling in the time domain. This is in fact the easiest and fastest way to perform a real time implementation of the convolution. However, real systems are time discrete. Thus, an exact shifting operation can only be done if the shifting time is a multiple of the digital step. This problem was solved in [15] by fixing the time between the impulses and changing only the magnitudes. Additionally, Murphy and Watanable discussed in [16] the design of an arbitrary rate digital shaping filter in the $z$-plane. Tuttle and Seering presented in [17] a systematic design tool to compute digital shapers using a discrete- time domain pole placement technique.

One severe drawback of Input shaping is that it optimizes the system response only after applying the last impulse. The system step response will have no zero control error before the time instant of the last impulse and can even present huge deviations from the reference signals. In this paper we present a systematic design tool to generate optimal impulse sequences for systems with long sampling period, that compensate totally any ramp time delay and minimize the quadratic control error that occurs before applying the last impulse. The theoretical results are applied to control the compliant end-effector of fig. 2.

\section{INPUT SHAPING}

In this section we give a brief review of the conventional input shaping techniques. The original method has been primarily developed for linear second order systems with the transfer function

$$
G(s)=\frac{y(s)}{u(s)}=K \frac{\omega_{0}^{2}}{s^{2}+2 D \omega_{0} s+\omega_{0}^{2}}
$$

with a static gain $K$, natural frequency $\omega_{0}$ and positive damping ratio $D$ smaller than 1 . The system has then the damped natural frequency

$$
\omega_{d}=\omega_{0} \sqrt{1-D^{2}}
$$

$u(s)$ and $y(s)$ denote respectively the system input and output.

It's known that applying an impulse $A_{0} \delta\left(t-t_{0}\right)$ to such a plant will result in an oscillating response $y_{0}(t)$. However a well chosen second impulse $A_{1} \delta\left(t-t_{1}\right)$ can excite a second oscillation $y_{1}(t)$ that totally cancels the first one (fig. 3). This idea can be extended to an impulse sequence with $n$ impulses

$$
f_{\delta}(t)=\sum_{i=0}^{n-1} A_{i} \delta\left(t-t_{i}\right) ; \quad t_{i}<t_{i+1}
$$

which compensates any oscillation immediately after applying the last impulse ([1]). By convolving this sequence with any desired command signal, new control inputs are generated which move the system without vibration. This command generation process is called input shaping. To design such a shaping filter we need consequently to derive the amplitudes $A_{i}$ and time instants $t_{i}$ with $i \in\{0,1, \ldots, n-1\}$.

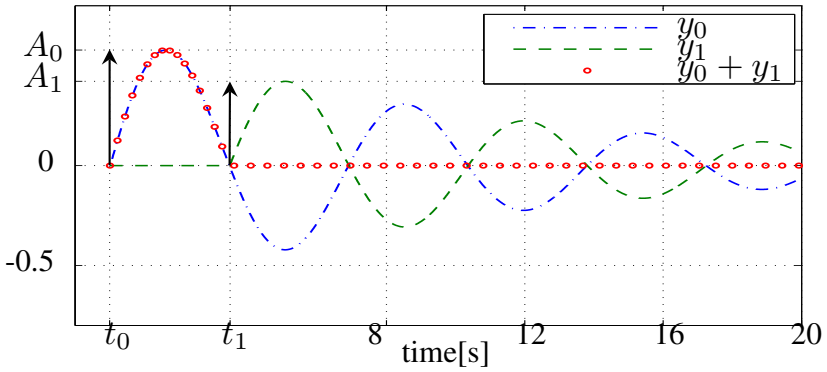

Fig. 3. Vibration cancellation when applying 2 impulses with: $\omega_{0}=1 s^{-1}$ and $D=0.1$.

The unity gain impulse response of (1) is:

$$
y(t)=\frac{\omega_{0}}{\sqrt{1-D^{2}}} e^{-\omega_{0} D t} \sin \left(\omega_{d} t\right) ; \quad t>0
$$

When applying the whole sequence (3) as an input to (1), then the response is the convolution result of (3) with (4). This can be understood as a linear combination of the delayed signals $y\left(t-t_{i}\right)$. Let $Y_{I S}(t)$ be the system response to (3) for $t \geq t_{n-1}$, then:

$$
\begin{aligned}
Y_{I S}(t) & =f_{\delta}(t) * y(t)=\sum_{i=0}^{n-1} A_{i} y\left(t-t_{i}\right) \\
& =\mathcal{A}\left(\omega_{0}, D\right) e^{-\omega_{0} D t} \sin \left(\omega_{d} t+\phi\right) ; \quad \phi \in \mathbb{R}(5)
\end{aligned}
$$

where:

$$
\begin{aligned}
& \mathcal{A}\left(\omega_{0}, D\right)=\omega_{0} \sqrt{\frac{\mathcal{C}^{2}\left(\omega_{0}, D\right)+\mathcal{S}^{2}\left(\omega_{0}, D\right)}{1-D^{2}}} \\
& \mathcal{C}\left(\omega_{0}, D\right)=\sum_{i=0}^{n-1} A_{i} e^{\omega_{0} D t_{i}} \cos \left(\omega_{d} t_{i}\right) \\
& \mathcal{S}\left(\omega_{0}, D\right)=\sum_{i=0}^{n-1} A_{i} e^{\omega_{0} D t_{i}} \sin \left(\omega_{d} t_{i}\right)
\end{aligned}
$$

Equation (6) tells us how strong the residual vibration will be for $t \geq t_{n-1}$. So, by setting $\mathcal{A}$ to zero, we enforce the response not to oscillate. This means that the responses $y_{i}$ of the respective impulses $A_{i} \delta\left(t-t_{i}\right)$ cancel each other immediately after the application of the last impulse. This is true if both of the squared terms in (6) are zero:

$$
\begin{aligned}
& \mathcal{C}\left(\omega_{0}, D\right)=0 \\
& \mathcal{S}\left(\omega_{0}, D\right)=0
\end{aligned}
$$

The filter will have a unity static gain if:

$$
\sum_{i=0}^{n-1} A_{i}=1 \text {. }
$$

If we additionally require that all the amplitudes $A_{i}$ are positive then we ensure that the maximum value of the convolution never exceeds the maximum value of the reference signal. The steady state value of the command and reference signal will be the same. Thus, the new input will never saturate the actuators if the original one does not. However 
requiring positive amplitudes is a relatively restrictive constraint that lead in general to long sequences of impulses. If fast responses are needed then it's recommendable to set constraints to the actuators's limitations and then solve for positive and negative amplitudes that satisfy them (Singhose [11]). A general form of these constraints is:

$$
\begin{aligned}
A_{i_{\min }} & \leq A_{i} \leq A_{i_{\max }} \\
\Delta A_{i_{\min }} & \leq \Delta A_{i} \leq \Delta A_{i_{\max }}
\end{aligned}
$$

Thereby $A_{i_{\min / \max }}$ and $\Delta A_{i_{\min / \max }}$ are the respective min$\mathrm{imal} /$ maximal allowed amplitude and increments values.

Some robustness has to be included into the design if exact estimations for $\omega_{0}$ and $D$ are not available. That is, the input shaper should still perform well even if the estimations of the plant parameters are not that good. For a first order robustness the derivative of (7) and (8) with respect to $\omega_{0}$ are constrained to zero (Singer and Seering [12]).

$$
\begin{aligned}
& \sum_{i=0}^{n-1} A_{i} t_{i} e^{\omega_{0} D t_{i}} \cos \left(\omega_{d} t_{i}\right)=0 \\
& \sum_{i=0}^{n-1} A_{i} t_{i} e^{\omega_{0} D t_{i}} \sin \left(\omega_{d} t_{i}\right)=0
\end{aligned}
$$

Equations (9), (10), (11), (14), (15) and the restriction (12), (13) define a constrained set of nonlinear equations (CSNE) that can be numerically solved for amplitudes $A_{i}$ and time instants $t_{i}$ to get a zero vibration robust input shaper.

When executing assembly tasks, the robot of fig 1 has to perform motions between several known locations in the Cartesian space. Those maneuvers are most of the time done by commanding positional ramps. Nevertheless, applying the conventional input shaping to a ramp results in additive time delay (fig. 4). This can lead to system performance degradation and even to instability if input shaping is used within a closed loop control scheme. Kapila, Tzes and Yan [13] presented in this context a closed loop control design for input shaped flexible structures using Lyapunov based stability. However including time delay compensation to the shaper design can improve this control scheme and is consequently strongly recommended. In the following section we show how an input shaper can be designed to compensate not only it's own time delay but the one caused by the dynamics too.

\section{RAmp Time Delay COMPENSATION}

Let $\tau$ be the ramp response time delay when applying input shaping (fig. 4). In order to be able to compensate it, we have first of all to figure out its relation with the input shaper parameters and the plant parameters. The unity gain step response $s(t)$ of (1) is

$s(t)=\left\{\begin{array}{l}1-\frac{1}{\sqrt{1-D^{2}}} e^{-\omega_{0} D t} \sin \left(\omega_{d} t+\varphi\right) \quad \text { for } t \geq 0 \\ 0 \text { otherwise }\end{array}\right.$

where

$$
\varphi=\arccos (D)
$$

Since the system (1) is linear, then the system response to a ramp is the time integral of the step response:

$$
\begin{aligned}
r(t) & =\int_{0}^{t} s(\xi) d \xi \\
& =t+\frac{e^{-\omega_{0} D t}}{\omega_{d}} \sin \left(\omega_{d} t+2 \varphi\right)-\frac{2 D}{\omega_{0}}
\end{aligned}
$$

Therefore:

$$
\lim _{t \rightarrow \infty}(t-r(t))=\frac{2 D}{\omega_{0}}
$$

Equation (19) gives the ramp response time delay for any

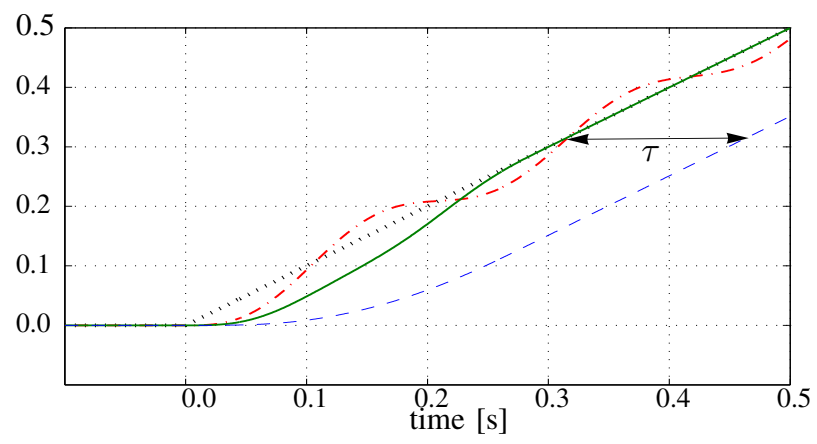

Fig. 4. Time delay compensation for $\omega_{0}=30 \frac{\mathrm{rad}}{\mathrm{s}}$ and $D=0.02$. dotted: reference signal. dashed-dotted: ramp response of system (1) dashed: system response with conventional input shaping. continuous: system response with time delay compensating input shaping.

linear second order oscillating plant. If the system is poorly damped then (19) will be insignificant which means that the main time delay is not caused by the dynamics but by input shaping. The plant response $r_{I S}(t)$ to a shaped ramp can be computed in analogy to (5). Here we consider the response after applying the last impulse $\left(t \geq t_{n-1}\right)$ :

$$
\begin{aligned}
r_{I S}(t) & =\left(t-\frac{2 D}{\omega_{0}}\right) \underbrace{\sum_{i=0}^{n-1} A_{i}}_{(10) \Rightarrow=1}-\sum_{i=0}^{n-1} A_{i} t_{i} \\
& -\frac{e^{-\omega_{0} D t}}{\omega_{d}} \cos \left(\omega_{d} t+2 \varphi\right) \underbrace{\sum_{i=0}^{n-1} A_{i} e^{\omega_{0} D t_{i}} \sin \left(\omega_{d} t_{i}\right)}_{(9) \Rightarrow=0} \\
& +\frac{e^{-\omega_{0} D t}}{\omega_{d}} \sin \left(\omega_{d} t+2 \varphi\right) \underbrace{\sum_{i=0}^{n-1} A_{i} e^{\omega_{0} D t_{i}} \cos \left(\omega_{d} t_{i}\right)}_{(8) \Rightarrow=0} \\
& =t-\frac{2 D}{\omega_{0}}-\sum_{i=0}^{n-1} A_{i} t_{i}
\end{aligned}
$$

Hence, the time delay resulting from the dynamics and input shaping is:

$$
t-r_{I S}(t)=\frac{2 D}{\omega_{0}}+\sum_{i=0}^{n-1} A_{i} t_{i}=\tau
$$


Notice that $\tau$ depends directly on the filter parameters. By setting it to zero, the dead time will be totally compensated. We have then:

$$
\sum_{i=0}^{n-1} A_{i} t_{i}=-\frac{2 D}{\omega_{0}}
$$

Including this feature to the filter design can be done by adding (22) to the CSNE as an additional equation. However requiring a total dead-time elimination leads often to huge amplitude values within short sequences of impulses. This can be avoided either by lengthening the sequence or by using predictive path scheduling within a known time delay (backward time shifting): When the trajectory is a priori known, then the command signals may be time advanced. This is valid for the experimental part of this paper. In fact, for a given assembly task the respective path structure is known. Any deviation from the manipulated objects is corrected predictively using camera data (Lange and Hirzinger [14]). In this case, (21) is used to enforce some known time delay $\tau_{0}$ which can be compensated due to command shifting:

$$
\sum_{i=0}^{n-1} A_{i} t_{i}=\tau_{0}-\frac{2 D}{\omega_{0}}
$$

To do so, the shifting time $\tau_{0}$ has to be a multiple of the sampling period. The same is also required for the time instants $t_{i}$ if the convolution is interpreted as time shifting and scaling operations. These both conditions are very serious implementation matters if the sampling period is a long one. Compared to the sampling rate of modern robotic system $(f=1 \mathrm{kHz})$, the sampling frequency of the used robot is much lower $(f=83 \mathrm{~Hz})$. In the following section, we describe a digital filter based on the ideas in [15]. The computational framework is arbitrarily extendable to any new constraints and keeps the length of the impulse sequence to a minimum.

\section{InPUT ShAPING FOR LOW SAMPLED SySTEMS}

In order to fit the time instants of the impulses to the sampling period $T=1 / f$ we can explicitly constrain all $t_{i}$ and $\tau_{0}$ to be a multiple of $T$ :

$$
t_{i}=z_{i} T \quad ; \quad \tau_{0}=m T
$$

Where:

$$
\begin{aligned}
z_{i} \in \mathbb{N} & ; m \in \mathbb{N} \\
z_{0} & =0 \\
z_{i} & <z_{i+1}
\end{aligned}
$$

$m$ is a design parameter used to set the ramp time delay to a known value. Equation (26) is used to constrain the first time instant $t_{0}$ to zero and have hence the fastest response. Adding (24) to the CSNE eliminates the time instants and replaces them by the integers $z_{i}$. The problem we want to solve can now be stated explicitly: Solve for $A_{i} \in \mathbb{R}$ and $z_{i} \in \mathbb{N}$ so that the equations (9), (10), (11), (14), (15), and (23) are satisfied under restriction of (12), (13), (26) and (27).
This formulation corresponds to a mixed-integer nonlinear problem (MINLP) which can be solved with a wide variety of commercial tools. Nevertheless, this implies the use of complex time- and resources consuming algorithms which consequently cannot be applied within online applications. To reduce the computation time, we can fix all $z_{i}$ so that they don't enter as unknown parameters in the problem any more. An adequate choice may be:

$$
z_{i}=i \quad t_{i}=i T
$$

This means that the $n$ impulses will be applied after each other with a time spacing of $T$. Notice that (28) already satisfies the constraints (26) and (27). Besides, it transforms the statements (9), (10), (11), (14), (15) and (23) from nonlinear to linear ones. This can be used to reformulate the equation set as following:

with $C=\left[\begin{array}{c}C \underline{A}=\underline{b} \underline{c}_{0}^{T} \\ \vdots \\ \underline{c}_{5}^{T}\end{array}\right] \in \mathbb{R}^{6 \times n} \quad ; \quad \underline{A} \in \mathbb{R}^{n} \quad ; \quad \underline{d} \in \mathbb{R}^{6}$

Where:

$$
\begin{aligned}
& c_{0, i}=e^{\omega_{0} D i T} \cos \left(\omega_{d} i T\right) \\
& c_{1, i}=e^{\omega_{0} D i T} \sin \left(\omega_{d} i T\right) \\
& c_{2, i}=1 \\
& c_{3, i}=i e^{\omega_{0} D i T} \cos \left(\omega_{d} i T\right) \\
& c_{4, i}=i e^{\omega_{0} D i T} \sin \left(\omega_{d} i T\right) \\
& c_{5, i}=i
\end{aligned}
$$

And:

$$
\begin{aligned}
& \underline{A}=\left[\begin{array}{llll}
A_{0} & A_{1} & \cdots & A_{n-1}
\end{array}\right]^{T} \\
& \underline{b}=\left[\begin{array}{llllll}
0 & 0 & 1 & 0 & 0 & m-\frac{2 D}{\omega_{0} T}
\end{array}\right]^{T} \\
& \text { (9) (10) (11) (14) (15) (23)(24)(28) }
\end{aligned}
$$

Notice that for $n \geq 6$, the equations (9), (10), (11), (14), (15), and (23) are linearly independent. Thus, $\operatorname{rank}(C)=6$.

The problem can now be reformulated as following: Find a vector of amplitudes $\underline{A}$ that satisfies (29), (12) and (13). For $n=6$, we have as much unknowns as equations. Thus, the unique solution is $\underline{A}=C^{-1} \underline{b}$. However we cannot expect that the constraints (12) and (13) are held. In general, this can be achieved within more than 6 degrees of freedom. In this case, the statement (29) is under-determined. This means that the matrix $C$ can not be inverted anymore since it's not quadratic. We have consequently for a given sequence length $n$ an infinity of solutions from which we need to select those that satisfy (12) and (13) if they exist. This task can be solved by many numerical iterative tools. Another practical and sometimes less time consuming alternative is to minimize the norm of the amplitudes $\underline{A}$ for a given sequence length $n$ and then check whether the constraints are satisfied. The following quadratic minimization problem is hence to be solved:

$$
\min \frac{1}{2}\|\underline{A}\|^{2}=\frac{1}{2} \underline{A}^{T} \underline{A} \quad \text { subject to } C \underline{A}=\underline{b}
$$


To proceed in solving (30) in an analytical way, we introduce the respective Lagrangian function:

$$
L(\underline{A}, \underline{\lambda})=\frac{1}{2} \underline{A}^{T} \underline{A}+\underline{\lambda}^{T}(C \underline{A}-\underline{b})
$$

Where $\underline{\lambda} \in \mathbb{R}^{6}$ is the vector of the Lagrange multipliers. Candidates for the minimum must satisfy:

$$
\left.\begin{array}{l}
\frac{\partial L}{\partial A}=\underline{A}+C^{T} \underline{\lambda}=0 \\
\frac{\partial \underline{L}}{\partial \underline{\lambda}}=C \underline{A}-\underline{b}=0
\end{array}\right\} \Rightarrow \underline{A}=C^{T}\left(C C^{T}\right)^{-1} \underline{b}
$$

Note that $C C^{T}$ is a $6 \times 6$ quadratic matrix with $\operatorname{rank}\left(C C^{T}\right)=6$ which makes it invertible.

Starting from $n=6$ we can solve for the optimal amplitude vector iteratively till the constraints (12) and

\begin{tabular}{|c|c|}
\hline \multicolumn{2}{|l|}{$n=6$} \\
\hline \multicolumn{2}{|c|}{ for(;;) } \\
\hline & Build $C$ and $\underline{b}$ \\
\hline & Compute $\underline{A}=C^{T}\left(C C^{T}\right)^{-1} \underline{b}$ \\
\hline & $\begin{array}{c}\text { (12) and (13) } \\
\text { satisfied? }\end{array}$ \\
\hline & $n=n+1$ \\
\hline
\end{tabular}
(13) are held (fig. 5). The number of the needed matrix

Fig. 5. Iterative scheme to compute an adequate sequence of impulses.

inversion operations increases linearly with the length of the sequence. Hence, the computational effort will be high for long sequences of impulses. This problem can be fixed by setting a better initial value for $n$ to reduce the number of iterations. In fact, this is always the case when the optimization is performed online: For a little variation of the plant parameters $\omega_{0}$ and $D$, the resulting new optimal sequence will have almost the same length as the old one. We can then initialize $n$ with the length of the old sequence. In this case we may extend the algorithm stated in fig. 5 to do not only forward but also backward constraints check. This means, if the constraints are already satisfied for an initial guess $n_{0}$, then we check whether they are also held for a sequence of length $n_{0}-1$. That way, we avoid operating with longer sequences than needed.

Choosing the amplitude vector $\underline{A}$ with the smallest norm results in smooth shaped commands and improves the shaper performance. However we still have no clear idea about what is happening with the system response for $t<t_{n-1}$. When a shaped step is applied to (1), the steady state is reached first at $t=t_{n-1}$. For long sequences of impulses the setting phase takes quite much time and should consequently be optimized. In the following section we present a design scheme, that satisfies all constraints introduced in the previous sections and minimizes the quadratic step response control error for $t<t_{n-1}$

\section{QuAdratic CONTROL ERROR Minimization}

Let $e(t)$ be the control error observed when a shaped unit step is applied to (1). Using (28), the shaped response will be:

$$
s_{I S}(t)=s(t) * \sum_{i=0}^{n-1} A_{i} \delta\left(t-t_{i}\right)=\sum_{i=0}^{n-1} A_{i} s(t-i T)
$$

Where $s(t)$ is the step response introduced in (16). For $t \geq t_{n-1}$, the steady state is reached and the vibrations are eliminated. At this point we suppose that a predictive path generator is available which accelerates the system response by shifting the inputs with $m T$ backward in the time. Hence:

$$
e(t)= \begin{cases}s_{I S}(t) & \text { for } t \in[0 ; m T] \\ 1-s_{I S}(t) & \text { for } t \in\left(m T ; t_{n-1}\right] \\ 0 & \text { otherwise }\end{cases}
$$

This control error can be minimized by using the time integral of the quadratic control error. Hence, the following objective function is introduced.

$$
\mathcal{I}_{q}=\underline{A}^{T} Q \underline{A}+\int_{0}^{\infty} e^{2}(t) d t
$$

where $Q$ is an $n \times n$ diagonal and positive definite weighting matrix. The larger the diagonal elements of $Q$ are, the severer are high amplitude values penalized. The minimization problem can now be formulated as following: Find the amplitude set $\underline{A}$ that minimizes (35) subject to (12), (13) and (29). Combining (28) and (34) yields:

$$
\begin{aligned}
& \int_{0}^{\infty} e^{2}(t) d t=\int_{0}^{m T} s_{I S}^{2}(t) d t+\int_{m T}^{(n-1) T}\left(1-s_{I S}(t)\right)^{2} d t \\
= & \underbrace{\int_{0}^{(n-1) T} s_{I S}^{2}(t) d t}_{\mathcal{I}_{q 2}}-2 \underbrace{\int_{m T}^{(n-1) T} s_{I S}(t) d t}_{\mathcal{I}_{q 1}}+(n-m-1) T
\end{aligned}
$$

One could already stop at this level and implement the quadratic control error term the way it is stated in (36). Then, an iterative numerical optimization tool is needed to minimize (35). However this leads to high computational effort since the integrals $\mathcal{I}_{q 1}$ and $\mathcal{I}_{q 2}$ have to be numerically evaluated for every call of the cost function (in every iteration). A better alternative is to proceed in simplifying $\mathcal{I}_{q 1 / q^{2}}$ and then solve analytically for the optimal amplitude vector $\underline{A}$ :

$$
\begin{aligned}
\mathcal{I}_{q 1} & =\int_{m T}^{(n-1) T} \sum_{i=0}^{n-1} A_{i} s(t-i T) d t \\
& =\sum_{i=0}^{n-1} A_{i} \underbrace{\int_{(m-i) T}^{(n-i-1) T}}_{\theta_{i}} s(t) d t=\underline{A}^{T} \underline{\theta}
\end{aligned}
$$


Where $\underline{\theta}$ is the vector composed of the elemental integrals $\theta_{i}$. The integration time span of $\theta_{i}$ can be reduced knowing that the step response $s(t)$ is zero for $t \leq 0$ (see 16):

$$
\theta_{i}=\int_{\max ((m-i), 0) T}^{(n-i-1) T} s(t) d t
$$

$\mathcal{I}_{q 2}$ can be evaluated in a similar way :

$$
\begin{aligned}
\mathcal{I}_{q 2} & =\int_{0}^{(n-1) T}\left[\sum_{i=0}^{n-1} A_{i} s(t-i T)\right]^{2} d t \\
& =\sum_{i, j=0}^{n-1} A_{i} A_{j} \underbrace{\int_{0}^{(n-1) T} s(t-i T) s(t-j T) d t}_{\psi_{i, j}} \\
& =\underline{A}^{T} \Psi \underline{A}
\end{aligned}
$$

Where $\Psi$ is the $n \times n$ matrix composed of the integrals $\psi_{i, j}$. The shifted step response $s(t-i T)$ is zero for $t \leq i T$. Hence:

$$
\psi_{i, j}=\int_{\max (i, j) T}^{(n-1) T} s(t-i T) s(t-j T) d t
$$

Notice that:

- $\psi_{i, j}=\psi_{j, i} \Rightarrow \Psi=\Psi^{T}$

- $\psi_{n-1, *}=\psi_{*, n-1}=0 \Rightarrow \operatorname{rank}(\Psi)<n$

The integrals $\theta_{i}$ and $\psi_{i, j}$ can now be computed either numerically or analytically and then used to compute $\mathcal{I}_{q 1}$, $\mathcal{I}_{q 2}$ and finally $\mathcal{I}_{q}$ :

$$
\mathcal{I}_{q}=\underline{A}^{T} \underbrace{(Q+\Psi)}_{\tilde{\Psi}} \underline{A}-2 \underline{A}^{T} \underline{\theta}+(n-m-1) T
$$

Equation (41) defines a linear quadratic cost function that has now to be minimized under the constraint (29). The solution can be computed using an appropriate Lagrangian function (see section IV). The resulting candidate for the minimum is:

$$
\underline{A}=\tilde{\Psi}^{-1}\left[\underline{\theta}-C^{T}\left(C \tilde{\Psi}^{-1} C^{T}\right)^{-1}\left(C \tilde{\Psi}^{-1} \underline{\theta}-\underline{b}\right)\right]
$$

Though $\Psi$ is singular, the matrix $Q$ can always be chosen so that $\tilde{\Psi}$ is a full rank matrix. Hence, $C \tilde{\Psi}^{-1} C^{T} \in \mathbb{R}^{6 \times 6}$ is regular and can be inverted too. Notice that the cost function (35) is maximized within infinite values of the amplitudes $A_{i}$. If $\tilde{\Psi}$ is a regular matrix then the solution vector stated in (42) will have only finite elements. Thus, (42) describes a minimum. To incorporate the actuator constraints (12) and (13) in the new design we may use the algorithm of fig 5. The only difference to section IV is that $\underline{A}$ is computed using (42) and not (32) any more. Simulation results showed that the new optimal step response presents a clearly better undelayed tracking behavior in the setting phase. For moderate diagonal values of $Q\left(q_{i}=0.2\right)$ and a set of 36 impulses, the cost function $\mathcal{I}_{q}$ could be reduced to $40 \%$ (fig. 6).

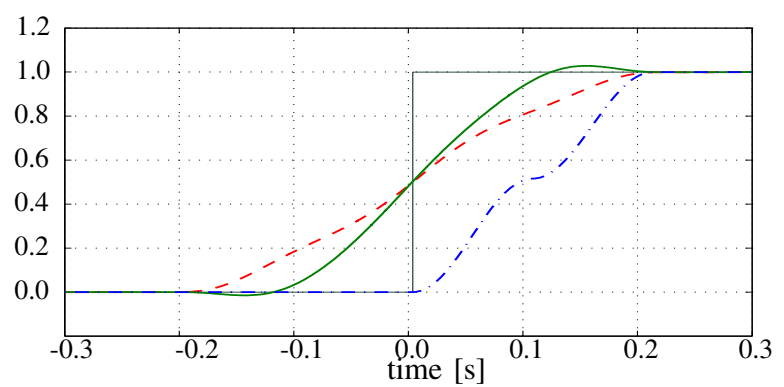

(a) Step responses

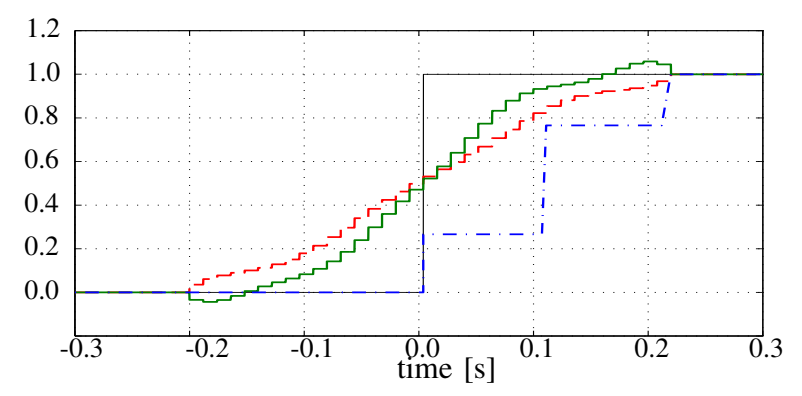

(b) outputs of the input shapers

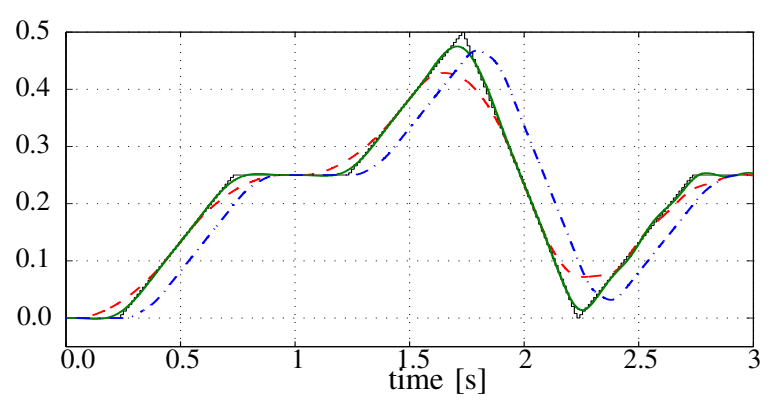

(c) System responses to a finite rate reference

Fig. 6. A representative sample of the simulation results for $\omega_{0}=30 \frac{\mathrm{rad}}{\mathrm{s}}$, $D=0.02, n=36, m=17, T=12 m s$ and $Q=0.2 I$. Continuous-thin: unshifted reference signals. Dashed: response of (1) without control error minimization (section IV). Continuous-thick: system response with control error minimization (section V). Dashed-dotted: response using conventional robust input shaping ( 3 impulses) which is not suitable for sampled systems

\section{EXPERIMENTS}

The presented input shaping control scheme is applied to the flexible end-effector of an industrial robot (KUKA KR180) with $T=12 \mathrm{~ms}$ when performing the task stated in fig. 1. The end-effector compliance is dominant with respect to the flexibility of the robot itself. So, the deflection of the force/torque sensor proves to be sufficient for the determination of the pose of the tool center point. Moreover, the compliance is concentrated within the mounting of the tool and can thus be treated in Cartesian space (fig. 2). In addition to the springs that are visible in fig. 2, the sensor comprises elastomers which offer both, elastic and damping characteristics. Unfortunately this is associated with a nonlinear behavior, in particular hysteresis which was compensated by a low gain integral controller. Deflections induced by gravitational forces are more important and have 
been considered by a lookup table with the end-effector orientation as entry.

The remaining end-effector dynamics are then identified using the linear time invariant model (1). The commands to the position controlled robot and the sensor measured deflections are considered to be respectively the process input and output. The experimental identification shows that several flexible modes of oscillations and couplings between the individual Cartesian directions exist. This is explained by the fact that the center of gravity and the compliance center do not coincide. Theoretically this has to be dealt by sequences of input shaping filters. In practice however, a single robust filter is sufficient for each direction since the parameters are quite similar: The natural frequency $\omega_{0}$ is varying between $25 \frac{\mathrm{rad}}{\mathrm{s}}$ and $28 \frac{\mathrm{rad}}{\mathrm{s}}$, while the damping ratio $D$ is between 0.02 and 0.04 . The current implementation of input shaping included only off line identification of the parameters. However an online version is planned in future works. Fig. 7 shows examples of the profit achieved by input shaping. These experiments show that a damping of $50 \%$

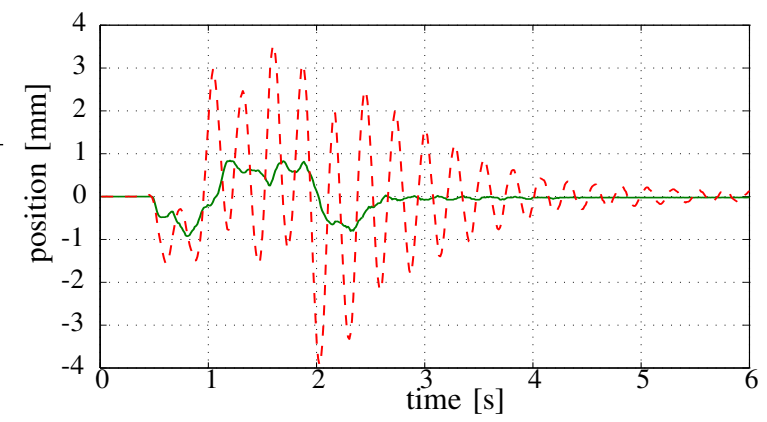

(a) Vibrations occurring in the Cartesian direction $y$ when applying a positional pulse in the Cartesian direction $x$.

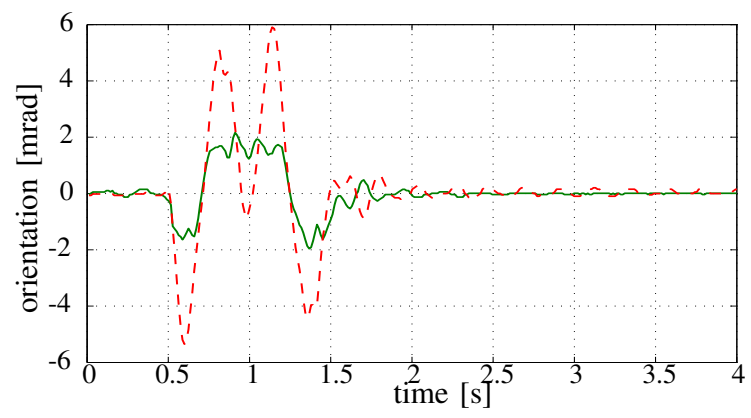

(b) Vibrations occurring in the Cartesian orientation $\alpha$ when applying an orientational pulse in the Cartesian orientation $\alpha$

Fig. 7. Sample of the experimental results. (a) Vibration damping within cross-coupled dynamics. (b) Vibration damping within direct dynamics. Dashed: output deflection without input shaping. Continuous: output deflection with the presented type of input shaping

is always reached in direct and cross-coupled oscillations although we use a unique filter for each Cartesian direction/orientation.

\section{CONCLUSION}

The paper demonstrates that the well known method of input shaping can be modified to fit to the long sampling periods of today's industrial robots. Since fixed robot paths can be commanded in advance, the resulting time delay is not unfavorable. Besides, intermediate control errors are minimized. It is worth mentioning that feedforward control methods as input shaping are inherently stable, even if the assumed process parameters are not appropriate. The only requirement is a stable position controller which is provided by the robot manufacturer. Future works will study the effects of the proposed method in the frequency domain.

\section{ACKNOWLEDGMENTS}

The authors would like to thank the Bayerische Forschungsstiftung for supporting this work.

\section{REFERENCES}

[1] Smith, O. J. M., "Posicast Control of Damped Oscillatory Systems" Proc. of the IRE, pp. 1249-1255, 1957.

[2] Singer, N. C., and Seering, W.P., "Preshaping Command Inputs to Reduce System Vibration", ASME J. of Dynamic Systems, Measurement and Control, Vol. 112, pp. 76-82, 1990.

[3] Singhose, W. E., Seering, W. P. and Singer, N. C., "Input Shaping for Vibration Reduction with Specified Insensitivity to Modeling Errors", Japan-USA Sym. on Flexible Automation, Boston, MA, 1996.

[4] Tzes, A. and Yurkovich, S., "An Adaptive Input Shaping Control Scheme for Vibration Suppression in Slewing Flexible Structures", IEEE Transactions on Control Systems Technology, Vol.1, 1993.

[5] Magee, D. P. and Book, W. J., "Filtering Micro-Manipulator Wrist Commands to Prevent Flexible Base Motion", American Control Conference, Seattle, WA, pp.924-928, 1995.

[6] Rhim, S., and Book, W., "Adaptive Time-delay Command Shaping Filter for Flexible Manipulator Control", IEEE/ASME Transactions on Mechatronics, Vol. 9, pp. 619-626, 2004.

[7] Singer, N. C., Singhose, W. E., and Seering, W. P., "Comparison of Filtering Methods for Reducing Residual Vibration", European Journal of Control, pp. 208-218, 1999.

[8] Robertson, M., Kozak, K., Singhose, W., "Computational Framework for Digital Input Shapers Using Linear Optimization," IEE Control Theory and Applications, Vol. 153, pp. 314-322, 2006.

[9] Tuttle, T., Seering, W., "Experimental Verification of Vibration Reduction in Flexible Spacecraft Using Input Shaping", Journal of Guidance, Control, and Dynamics, Vol. 20, pp. 658-664, 1997.

[10] Singhose, W. E., Kim, D., "Manipulation with Tower Cranes Exhibiting Double-Pendulum Oscillations", IEEE International Conference on Robotics \& Automation (ICRA), Roma, Italy, pp. 4550-4555, April 2007.

[11] Singhose, W. E., Seering, W. P., Singer N. C., "Time-optimal negative input shapers" J. of Dynamic Systems, Measurement, and Control, 1997

[12] Singer, N. C., Seering, W. P., "Preshaping Command Inputs to Reduce System Vibration",Journal of Dynamic Systems, Measurement, and Control, Vol. 112, pp. 76-82, 1990.

[13] Kapila, V., Tzes, A., Yan Q., "Closed-Loop Input Shaping for Flexible Structures using Time-Delay Control", Proceedings of the 38th Conference on Decision \& Control, Phoenix, Arizona, USA, December 1999.

[14] Lange, F., Hirzinger, G., "Spatial Vision-Based Control of High-Speed Robot Arms" Industrial Robotics: Theory, Modeling and Control, Advanced Robotic Systems, Vienna, Austria, 2007.

[15] Singer, N. C., "Residual vibration reduction in computer controlled machines", Ph.D. dissertation, AI-TR 1030, Artificial Intell. Lab., MIT, Cambridge, MA, January 1989.

[16] Murphy, B. R., Watanabe, I., "Digital Shaping Filters for Reducing Machine Vibration" IEEE Transactions on Robotics and Automation, Vol. 8, pp. 285-289, 1992.

[17] Tuttle, T., Seering, W., "A Zero-placement Technique for Designing Shaped Inputs to Suppress Multiple-mode Vibration," American Control Conference, Baltimore, MD, 1994, pp. 2533-2537. 